\title{
Perú: La consejería balanceada aumenta la calidad de atención y el conocimiento de las mujeres sobre anticonceptivos
}

Frontiers in Reproductive Health

Follow this and additional works at: https://knowledgecommons.popcouncil.org/departments_sbsr-rh How does access to this work benefit you? Let us know!

\section{Recommended Citation}

"Perú: La consejería balanceada aumenta la calidad de atención y el conocimiento de las mujeres sobre anticonceptivos," FRONTERAS Resumen de Investigación Operativa. Ciudad de México: Population Council, 2000. 
Perú Calidad de la
atención

Investigación
operativa

Resumen 23

\section{La Consejería Balanceada aumenta la calidad de la atención y el conocimiento de las mujeres sobre anticonceptivos}

Cuando los proveedores mejoraron las sesiones de consejería con el uso de un algoritmo y materiales de apoyo, la tasa de uso de planificación familiar a 12 meses sólo aumentó ligeramente. Si bien este aumento tuvo un impacto práctico limitado, la intervención mejoró el comportamiento de algunos proveedores e incrementó el conocimiento de las clientas acerca del DIU y los métodos hormonales.

\section{Antecedentes}

Desde hace tiempo, existe la hipótesis de que una mejor calidad en la interacción clienta-proveedor (ICP)

incrementa el uso de métodos de planificación familiar y el conocimiento de las clientas al respecto; sin embargo, hay poca información que respalde esta hipótesis.

En el año 2000, el Ministerio de Salud del Perú (MINSA) solicitó la ayuda del Programa FRONTERAS para mejorar la calidad de la ICP en sus clínicas de planificación familiar. En una investigación previa (León et al. 1999) se observó que los proveedores daban demasiados detalles sobre todos los métodos disponibles, y muy pocos acerca del método elegido por la clienta. Como resultado, las clientas padecían, al parecer, de un exceso de información y un aprendizaje deficiente.

Para atender esta deficiencia, los investigadores probaron la "Estrategia de Consejería Balanceada", un algoritmo que se usa junto con un juego de tarjetas para ayudar a las clientas a elegir un método, y folletos con información sobre los métodos. Doce distritos de salud del Perú se asignaron de manera aleatoria a un grupo experimental, y 12 más a un grupo de control. Participaron 334 centros de salud y un total de 279 proveedores (alrededor de uno por centro) y 75 supervisores, quienes recibieron capacitación sobre el uso del algoritmo y los materiales de apoyo. Clientas simuladas y observadores registraron el comportamiento de los proveedores después de la capacitación. El uso de anticonceptivos, el logro de las metas reproductivas y los conocimientos de las clientas se midieron al terminar la intervención y 13 meses después.

\section{Hallazgos}

- La Estrategia de Consejería Balanceada mejoró el uso de planificación familiar a 12 meses en comparación con el grupo control. Sin embargo la diferencia fue poca en términos prácticos $(81 \%$ contra $78 \%)$. El estudio examinó la tasa de abandono de métodos atribuible a la calidad de la atención, como por ejemplo el abandono por efectos secundarios u otros inconvenientes. El uso continuo atribuible a la calidad de la atención, es decir, aquel que puede modificarse con intervenciones en los programas de servicios, fue significativamente más alto en el grupo experimental (ver la figura).

Tendencias en el uso de todos los métodos atribuible a la calidad de atención, grupo experimental y control

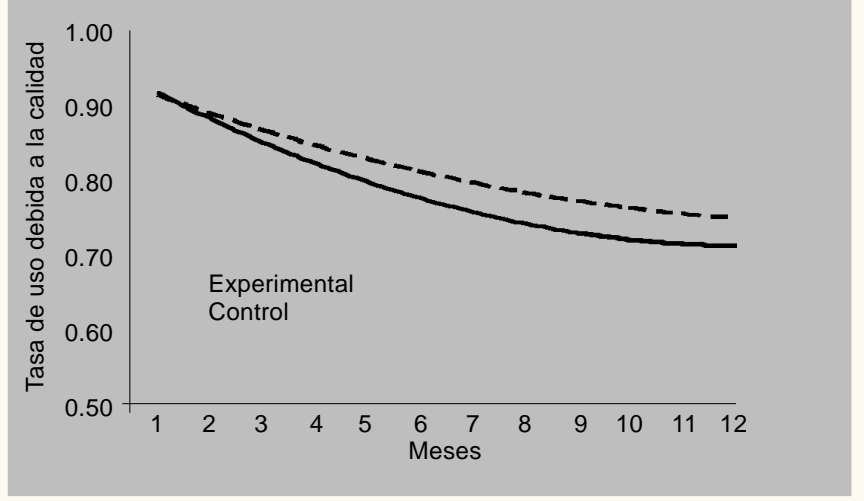




\section{Utilización}

- Generalmente, se considera que el objetivo más importante de un programa es satisfacer las intenciones reproductivas de la mujer. Este estudio observó hasta qué punto se cumplieron dichas intenciones durante un año. La intervención no logró mejorar el cumplimiento de las mismas cuando las mujeres las definieron durante el primer contacto con el programa.

- Trece meses después de la intervención, el conocimiento de las clientas sobre el DIU y los métodos hormonales era más alto en el grupo experimental (12.2 en promedio contra 10.4, en una escala de 18 puntos). No hubo diferencias significativas en el conocimiento de métodos naturales modernos ni de barrera (los métodos menos utilizados en el Perú).

- El uso de los materiales fue bajo. Sólo el 37 por ciento de los proveedores del grupo experimental utilizaron la Estrategia de Consejería Balanceada completa, es decir, tanto el algoritmo como las tarjetas y los folletos. Sólo cuando los proveedores utilizaron todos los componentes de la Estrategia, mejoró la calidad de atención y las clientas obtuvieron mayores conocimientos. En una prueba posterior de la Estrategia en Guatemala, se agregaron cuatro visitas de supervisión a la intervención; de este modo, el uso de los materiales aumentó a cerca del 70 por ciento de los proveedores (León et al. 2003).
- Hasta ahora, la Estrategia de Consejería Balanceada ha sido implementada en dos tipos de escenarios, con resultados notablemente distintos. Uno de ellos involucró pequeños centros de atención primaria en Perú y Guatemala. Los estudios en estos escenarios mostraron claras mejoras en la calidad de la atención. Sin embargo, al probar la estrategia en grandes hospitales en Perú y Guatemala, la evidencia de mejoras fue poca y algunos resultados fueron ambiguos o negativos.

\section{Implicaciones normativas}

- La Estrategia de Consejería Balanceada mejoró el comportamiento de las clientas en mayor o menor medida dependiendo de factores del programa distintos a la ICP misma. Las clientas del grupo experimental que seleccionaron el DIU o un método hormonal tenían mayor conocimiento sobre su método que las clientas del grupo de control, incluso cuando sólo una minoría de los proveedores cumplió con la Estrategia de Consejería Balanceada. Si bien no se encontraron diferencias importantes en el uso de métodos a 12 meses, la Estrategia ofrece un modelo para los programas que buscan mejorar la calidad de atención y el conocimiento de las clientas.

La Estrategia parece funcionar mejor en un contexto de atención primaria que en grandes hospitales, y se requieren cambios adicionales al programa, como una mejor supervisión, para optimizar el uso del algoritmo y los materiales de apoyo por parte de los proveedores.

El estudio es uno de tan sólo unos pocos experimentos para obtener evidencia de que los cambios en la calidad de la IPC pueden afectar significativamente el uso continuo de anticonceptivos.

Marzo 2000

\section{Fuentes:}

León, Federico R., Santiago Roca, Alex Ríos, Adriana Zumarán y Ana Rosa Feijóo. 2003. "One-year client impacts of quality of care improvements achieved in Peru [Impactos a un año de las mejoras en la calidad de la atención lograda en Perú]," FRONTIERS Final Report. Washington, DC: Population Council. Disponible en nuestro portal: http://www.popcouncil.org/pdfs/frontiers/FR FinalReports/ Peru CPI.pdf o por correo electrónico: frontiers@pcdc.org

León, Federico R., Alex Ríos, Adriana Zumarán, Marisela de la Cruz, Carlos Brambila y John H. Bratt. 2003. "Enhancing quality for clients: The balanced counseling strategy [Mejorar la calidad de atención para las clientas: La estrategia de consejería balanceada]," FRONTIERS Program Brief No. 3. Washington, DC: Population Council. Disponible en nuestro portal: http://www.popcouncil.org/pdfs/ frontiers/pbriefs/balance counseling brf.pdf o por correo electrónico: frontiers@pcdc.org

León, Federico R. 1999. "Peru: Providers' compliance with quality of care norms [Cumplimiento de las normas de calidad de atención en el Perú],” FRONTIERS Final Report. Washington, DC: Population Council. Disponible en nuestro portal: http://www.popcouncil.org/ pdfs/frontiers/FR FinalReports/peru qoc.pdf o por correo electrónico: frontiers@pcdc.org

Este proyecto se llevó a cabo con el apoyo de la AGENCIA DE LOS ESTADOS UNIDOS PARA EL DESARROLLO INTERNACIONAL (USAID) bajo el Acuerdo de Cooperación No. HRN-A-00-98-00012-00. 